differences lead to a different movement pattern of the affected arm. The effect of the OBPP on the level of activity and participation seems to be of no importance. The children with an OBPP do not stay behind with children of their age in physical and social activities.

Conclusions Children with a conservative treaded OBPP have persisting restrictions specially on the level of body functions and structure. Despite these restrictions there is no relevant effect shown on the activity and participation level. Using the mobility tests we think there is a relation between position of the scapula and the joint mobility.

\section{SMOKING AND OTHER PRE-GESTATIONAL RISK FACTORS FOR SPONTANEOUS PRETERM BIRTH}

doi:10.1136/archdischild-2012-302724.1318

1.2PF Freitas, ${ }^{3}$ E Grillo. ${ }^{1}$ Public Health, Universidade Federal de Santa Catarina; ${ }^{2}$ Curso de Medicina, Universidade do Sul de Santa Catarina UNISUL; ${ }^{3 P e d i a t r i a, ~ U n i v e r s i d a d e ~}$ Federal de Santa Catarina, Florianópolis, Brazil

Objectives To investigate pre-gestational risk factors for spontaneous preterm birth and, the role of smoking and its cumulative effects on prematurity.

Methods A case-control study analyzed all births $(2,198)$ occurring in a tertiary maternity hospital between April 2002 and July 2004. Spontaneous preterm births of single and live newborns without malformations were selected as cases. Controls were all the term births of live and single newborns without malformations during the same period. Three outcomes were studied: all preterm births ( $<37$ weeks), less than 35 weeks and less than 32 weeks of gestational age. Logistic regression was used to obtain the independent effect of pre-gestational risk factors.

Results Maternal age of less than 20 years, low schooling, low maternal pre-gestational body mass index and smoking showed significant, independent association with spontaneous preterm birth for the three outcomes. For all these risk factors, excepting maternal smoking, odds ratios increased with decreasing gestational age at birth and this trend was significant for low maternal age and low pre-gestational body mass index.

Conclusions The cumulative effects of smoking calls for the need to encourage smoking cessation among pregnant women, especially those who are underweight and in the older age groups, because of the increased risk of delivering premature babies.

\section{VITAMIN D LEVELS IN NEAR TERM INFANTS}

doi:10.1136/archdischild-2012-302724.1319

'W Al-Harthy, ${ }^{2} \mathrm{D}$ El-Metwaly, ${ }^{3} \mathrm{MM}$ Abou Al-Seoud. 'Neonatal Division, Paediatric Department, Maternity and Children Hospital, Makkah; Saudi Arabia; '2Paediatric, Suez Canal University (FOMSCU), Ismaillia, Egypt; ${ }^{3}$ Neonatal Division, Paediatric Department, King Fahad Armed Forces Hospital, Jeddah, Saudi Arabia

Background and Aims Based on the fact that the whole-body bone mineral content increases between 32-33 wk and 40-41 wk gestation (3.0 fold increase), near-term infants are thus at higher risk for low levels of vitamin D. Our aim was to measure 25-hydroxyvitamin D [25(OH) D] concentrations in the cord blood of near term infants born at KFAFH in Jeddah Saudi Arabia.

Methods This was a prospective study carried out from August $1^{\text {st }}$ 2011 to January $31^{\text {st }} 2012$. A total of 153 near terms babies with gestational ages 34 to $36+6$ weeks were included. The results were classified as Deficient $(25(\mathrm{OH}) \quad \mathrm{D}<37.5 \mathrm{nmol} / \mathrm{L})$, Insufficient $(25(\mathrm{OH}) \mathrm{D} 37.5-75 \mathrm{nmol} / \mathrm{L})$ or Sufficient $(25(\mathrm{OH}) \mathrm{D}>75 \mathrm{nmol} / \mathrm{L}]$.

Results The measured 25(OH) D levels ranged between 3-91 $\mathrm{nmol} / \mathrm{L}$ with a mean of $26.91 \pm 13.934 \mathrm{nmol} / \mathrm{L}$. The values were classified as deficient in 134 babies (88.2\%) and insufficient in 16 babies $(10.5 \%)$, while only two babies $(1.3 \%)$ had sufficient levels of vitamin $\mathrm{D}$ in their cord blood. We ran correlation studies between $25(\mathrm{OH})$ D levels, birth weight $(\mathrm{BWt})$, gestational age (GA) and admission to the neonatal intensive care (NICU), there was a weak correlation between $25(\mathrm{OH})$ D levels and birth weight $r$ value of. 172 $(p<0.05)$ but no correlation was found with GA or NICU admission.

Conclusion We observed a high prevalence of significant hypovitaminosis $\mathrm{D}$ among near term infants and a weak correlation with lower birth weight, the magnitude of which warrants identification and intervention.

\section{BIRTH AND DISCHARGE WEIGHT OF INFANTS WITH BIRTH WEIGHT LESS THAN $1000 \mathrm{G}$}

doi:10.1136/archdischild-2012-302724.1320

1J Tołloczko, 'A Góralska, ${ }^{2} \mathrm{~A}$ Borowiec, 'MK Kornacka. 'Department of Neonatology and Neonatal Intensive Care; ${ }^{2}$ Students Scientific Association of Neonatology, Medical University of Warsaw, Warsaw, Poland

Background and Aims Despite sweeping progress in the area of neonatology it is impossible to create artificial environment which would provide the same development conditions as mother's womb. Correct subsequent development of a child is dependant on intrauterine growth. Premature delivery hampers not only the development of internal organs but also weight gain. Aim of the project is to compare birth and discharge weight of infants with ELBW in order to analyse their weight gain.

Methods Data was gathered from 81 infants of weight less than $1000 \mathrm{~g}$ hospitalised in Neonatology and Infant Intensive Care Centre at the Medical University of Warsaw. Employing growth charts to compare birth and discharge weight of infants with ELBW. Assessing the influence of pregnancy length and sex of the infant on its weight gain.

Results The 81 infants were divided into 3 groups. Group 1 with 22 infants $(27.2 \%)$ whose weight-for-age score was the same for both birth and discharge weight. Group 2 with 53 infants (70.4\%) for which weight-for-age score lowered after birth. Group 3 with 6 infants $(2.4 \%)$ for which weight-for-age score increased. A statistically significant correlation was discovered between pregnancy length and weight gain rate in groups 1 and 2. No correlation was found between sex of the infant and its gain weight in any of the groups.

Conclusions For infants with ELBW postnatal weight gain does not match intrauterine growth. An important factor hampering postnatal weight gain is pregnancy length. The lower intrauterine age the slower weight gain rate.

\section{THE MANAGEMENT OF CENTRAL DIABETES INSIPIDUS IN NEONATAL INTENSIVE CARE UNIT: EXPERIENCE OF EIGHT CASES}

doi:10.1136/archdischild-2012-302724.1321

'S Alan, 'A Kılıç, 'U Çakır, 'D Yıldız, 'D Kahvecioğlu, ${ }^{2} \mathrm{M}$ Berberoğlu, ${ }^{2} Z$ Şıklar, ${ }^{2} \mathrm{G}$ Öçal, 'Ö Erdeve, 'B Atasay, 'S Arsan. 'Department of Pediatrics, Division of Neonatology; ${ }^{2}$ Department of Pediatrics, Division of Pediatric Endocrinology, Ankara University, Ankara, Turkey

Neonatal central diabetes insipidus (DI) is extremely rare and etiology has not been documented extensively. Asphyxia, intraventricular hemorrhage, severe infections, and central nervous system abnormalities have been associated with central DI in neonatal intensive care units (NICU). Desamino-8-D-arginine vasopressin (DDAVP) has been in clinical use for the treatment of central DI DDAVP preparations are available for intranasal, oral, subcutaneous, and intravenous administration. There is not clear data for the management and used DDAVP form for the central DI in neonates. In this article, we presented eight cases with different etiology of 
neonatal central DI. Six cases were preterm with intracranial hemorrhage and the other two patients were congenital toxoplasmosis one of which was preterm. All of the cases received oral desmopres$\sin$ at a dose of $10 \mathrm{mcg} / \mathrm{kg} /$ day. And then oral desmopressin dosage was adjusted according to the serum sodium and urine output. All cases were treated successfully with oral DDAVP. Three cases who have intracranial hemorrhage died due to other preterm complication. According to our case series, oral DDAVP is an applicable, safe and effective form of DDAVP.

\section{INTER-HOSPITAL TRANSPORT OF NEONATES IN THE SOUTH OF VIETNAM - RELATIONS TO PROGNOSIS AND OUTCOME}

doi:10.1136/archdischild-2012-302724.1322

${ }^{1} \mathrm{JIM}$ Andersen, 'MM Andersen, ${ }^{2} \mathrm{FK}$ Pedersen. 'University of Copenhagen; ${ }^{2}$ Pediatric Clinic, Juliane Marie Centeret, Rigshospitalet, Copenhagen, Denmark

Background and Aim To improve neonatal conditions in the South of Vietnam through mapping the conditions during interhospital transfer, revealing avoidable factors and unmasking cultural differences allowing action towards improvements.

Method A prospective and analytical cohort study was conducted at Pediatric Hospital \#1 in HCM City, Vietnam. Data was collected through standardized questionnaires and during a set timeframe in a one-month period. Infants $<28$ days or weighing $<2000 \mathrm{~g}$, transferred from the south-half of Vietnam were included giving a total of 103 neonates representing 96 transfers.

Results A transfer $>120$ min increased the risk of admission $>8$ days in total $(p=.027)$ and in NICU $(p=.001)$. The infants most vulnerable to transfer were more frequently transferred by an ambulance equipped and escorted to handle emergency than the least vulnerable $(\mathrm{p}=.003)$. However, the transfers of intermediate quality (51.5\%) had significantly worse outcomes ( $p=.007) .28 .2 \%$ of the ambulances were adequately equipped to handle emergencies and none had monitoring equipment for neonates. All neonates were escorted by health personnel, though none had transport training and only $32 \%$ had appropriate qualifications. Initiated treatment was continued, but no new initiatives were taken.

Conclusion The inter-hospital transport is of good standard, all transfers being by ambulance, with medical accompaniment and some pre-assessment made. A long transfer still worsens the outcome of the neonates significantly. However, our study reveals factors, which can be improved prior to, during and following transfer. In order to succeed with new approaches, the cultural aspects must be acknowledged.

\section{IMPACT OF THE NEAR TERM INFANTS ON NICU BED UTILIZATION}

doi:10.1136/archdischild-2012-302724.1323

EH Abdulrahman, MM Abou Al-Seoud. Neonatal Division-Paediatric Department, King Fahad Armed Forces Hospital, Jeddah, Saudi Arabia

Background Near term infants are prone to more medical problems than full term infants requiring more neonatal intensive careunit (NICU) admissions

Aim Study the impact of near term infants on the utilization of NICU beds.

Methods Retrospective collection of hospital newborn electronic database from January 2007 - December 2011.

Two groups were identified group 1 infants 37 weeks and above and group 2 near term infants 34 weeks- $36+6$ weeks. The following demographics collected, Birth weight (BWT), Gestational age (GA), gender, length of stay (LOS) and NICU admission.

Results The number of newborns delivered during the study period were41089. Near term infants were 3722 (9\%). Mean birth weight was $3.1 \pm 0.458 \mathrm{~kg}$ for group 1 and $2.42 \pm 497 \mathrm{~kg}$ for group 2 (p 0.0001). 5835 (14\%)infants required NICU admissions at birth in group 1 and 1025(28\%) in group 2 (p 0.09). LOS was calculated a mean of $2.58 \pm 3.33$ days in group 1 and $8.5 \pm 15.58$ days in group 2 (p.0001).

Using multiple regression analysis birth weight and male gender were predictors for prolonged hospital LOS and the need for NICU admission in both groups.

Conclusion In our population near term infants were more likely to utilize NICU beds for significantly longer LOS compared to full term which increases costs and limit availability of NICU beds in our community.

\section{CLINICAL PROFILE AND OUTCOME OF NEWBORNS WITH ACUTE KIDNEY INJURY IN A LEVEL 3 NEONATAL UNIT IN WESTERN INDIA}

doi:10.1136/archdischild-2012-302724.1324

AR Kungwani, AS Nimbalkar, AR Sethi, DV Patel, SM Nimbalkar. Department of Pediatrics, Pramukhswami Medical College, Anand, India

\section{Background and Aims Our study aims are}

1. To study the clinical profile and associated conditions in newborns with Acute Kidney Injury (AKI) in our Level 3 Neonatal ICU.

2. To evaluate the incidence and outcome of newborns with AKI.

3. To study risk factors associated with AKI in our newborns.

Methods AKI was defined as Serum Creatinine $>1.5 \mathrm{mg} / \mathrm{dl}$. Data about neonates admitted over period of three years were studied from a Level 3 Neonatal Intensive Care Unit in Western India. Parameters associated with AKI were evaluated and descriptive analysis was done.

Results Out of 1745 patients, 74 (Male-61, Female-13) patients had AKI. The incidence of AKI was $4.24 \%$ of admitted newborns, and $80 \%$ of the babies developed AKI within first week. Incidence of AKI in outborn babies (5.83\%) is higher than inborn babies $(2.44 \%)$ and outborns comprised $72.97 \%$ of all AKIs (54/74). Mean Birth weight of neonates was $2.46 \pm 0.55 \mathrm{~kg}$ with $47.30 \%$ being LBWI. Factors associated with AKI were sepsis (91.9\%), shock(64.9\%), requirement of ventilation $(62.2 \%)$, Perinatal asphyxia (36.5\%), resuscitation $(40.5 \%)$ and requirement of intubation at birth (23\%). Mean Serum Creatinine was $2.87 \pm 1.81 \mathrm{mg} / \mathrm{dl}(1.51$ to 10.05$)$. Mean age at diagnosis was $5.76 \pm 6.64$ days(1 to 41 ). The mortality was $20 \%$ and $51.6 \%$ patients went DAMA (Discharge Against Medical Advice).

Conclusions Mortality in patients with AKI is very high. Most AKI occurs in the first week of life and factors associated with AKI are easily recognizable and should prompt early referral of neonates.

\section{5 \\ INTRA-SUBJECT VARIABILITY ON 3T MRI IMAGING OF NEONATAL HIPS}

doi:10.1136/archdischild-2012-302724.1325

${ }^{1} \mathrm{H}$ Hill, ${ }^{2} \mathrm{~A}$ Murphy, ${ }^{3} \mathrm{E}$ Jacqz-Aigrain, ${ }^{4} \mathrm{LJ}$ Abernethy, ${ }^{1} \mathrm{MA}$ Turner, TINN (Treat INfections in Neonates). 'Women's and Chidren's Health, University of Liverpool; ${ }^{2}$ Neonatal Unit, Liverpool Women's NHS Foundation Trust, Liverpool, UK; ${ }^{3}$ Paediatric Pharmacology and Pharmacogenetics, Robert Debré Hospital - APHP, Paris, France; ${ }^{4}$ Clinical Radiology, Alder Hey NHS Foundation Trust, Liverpool, UK

Background MRI may have utility for examining neonatal hips. To date, MRI measurements of hip dimensions have only been characterized on post-mortem imaging. The aim of this study was to compare measurements made on left and right neonatal hips.

Methods Neonatal hips were imaged at 3T. Acetabular width and depth were measured using methods described by Whitby 